\title{
A Novel Method of Investigating Wet Bentonite Clay Particles in TEM
}

\author{
Yuma Kuwamura $^{1}$, Wen-An Chiou ${ }^{2}$, Hiroki Minoda ${ }^{1}$, Reiner Dohrmann ${ }^{3}$ and Stephan Kaufhold ${ }^{3}$ \\ 1. Department of Applied Physics, Tokyo University of Agriculture and Technology, Naka Cho, \\ Koganei, Tokyo 184-8588, Japan \\ 2. NISP Lab, NanoCenter, University of Maryland, College Park, MD 20742-2831, USA \\ 3. Federal Institute for Geosciences and Natural Resources, State Authority of Mining, Energy and \\ Geology, Geozentrum Hannover, Stilleweg 2, D-30655 Hannover, Germany
}

Over the last decade, much attention has been devoted to in-situ electron microscopy of nanomaterials due to the advantages of examining materials reaction in dynamic environments. Investigation of small particles of aluminum silicate (clay minerals) has been difficult due to the special properties of clay particles. Although special TEM holders for different in-situ TEM experiments have been developed and commercialized, the high cost of commercial products has prevented many researchers from pursuing in-situ TEM research. This paper presents a simple, costeffective method of constructing an environmental cell (EC) for in-situ wet TEM research.

Special carbon films were fabricated following Fukami's method [1]. Carbon films made by other conventional methods may also be used. A wet cell EC sealed with two carbon films was built to fit in a regular TEM sample holder (Fig. 1). To prevent possible breakage of carbon film, the hole that allows the electron beam to penetrate is rather small $(\sim 1 \mathrm{~mm})$. Bentonite, a special species of smectitic clay minerals that contain high $\mathrm{Mg}$ and Fe, collected from different localities was purified to remove organic matter and other impurities from the original soil. Bentonite particles were fully dispersed in deionized water and/or ethanol. A tiny drop of bentonite suspension was first pipetted onto the bottom side of grid, and then covered with another carbon grid on the top. A very small amount of vacuum grease was also applied to seal both grids. Special care was exercised to prevent adhesives from entering into the grids and contaminating the sample. The EC with wet sample was evacuated in a pre-pumping chamber to ensure the integrity of the wet cell before inserting into the TEM (modified JEOL JEM 2010 operated at $200 \mathrm{kV}$ ) equipped with a CCD camera for in situ study.

TEM images (Fig. 2) show typical morphology and distribution of bentonite clay particles in an airdried sample. Although clay particles can be well-dispersed in water or ethanol, clay particles, especially bentonite, prepared by the conventional technique often stick together and form large/thick aggregates when dry. At best, images from conventional TEM micrographs capture the gross platy, cloudy and/or fluffy nature of those clays [2], but discerning the size and shape of particle morphology in detail is nearly impossible. The real size and shape of very small clay particles may easily be obscured by large clay aggregates. However, using the newly developed wet EC, same bentonite clay particles were well-dispersed (Fig. 3). Particle sizes ranging from 20 to 200 $\mathrm{nm}$ are visible. The low contrast of those small particles indicates that bentonite particles are made of a very thin layer silicate oxide and probably floating in the EC (Fig. 3a). A variety of particle shapes can also been seen, though some particles are overlapped due to the high concentration of clay suspension (Fig. 3b). The clearly defined particle size and shape now allows for statistical particle size analysis to be performed. This unique method provides a simple method for preparing and investigating wet samples in TEM at low cost.

[1] A. Fukami and K. Adachi, Jour. Electron Microscopy. 14 (1965) p.112.

[2] R. E. Grim, "Clay Mineralogy", McGraw-Hill Book Co., (1963) 596p. 
[3] Part of TEM work performed at NISP Lab was partially supported by NSF-MRSEC (DMR 05-20471, Shared Experimental Facility) and UMD.

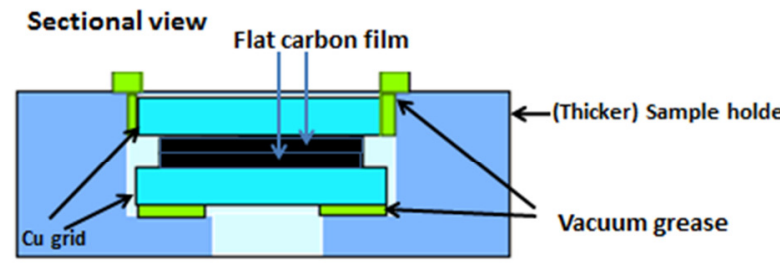

Cu grid: hole size $0.1 \mathrm{~mm}$ (diameter) hole number 19 (9) $3 \mathrm{~mm} \rightarrow$

About $\sim 1 \mathrm{~mm}$

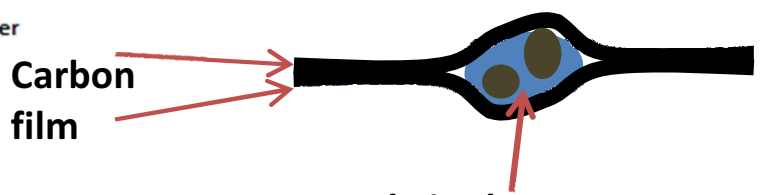

Sample in the water

Fig. 1. Schematic diagram showing basic concept (right) and construction (left) of the wet EC.

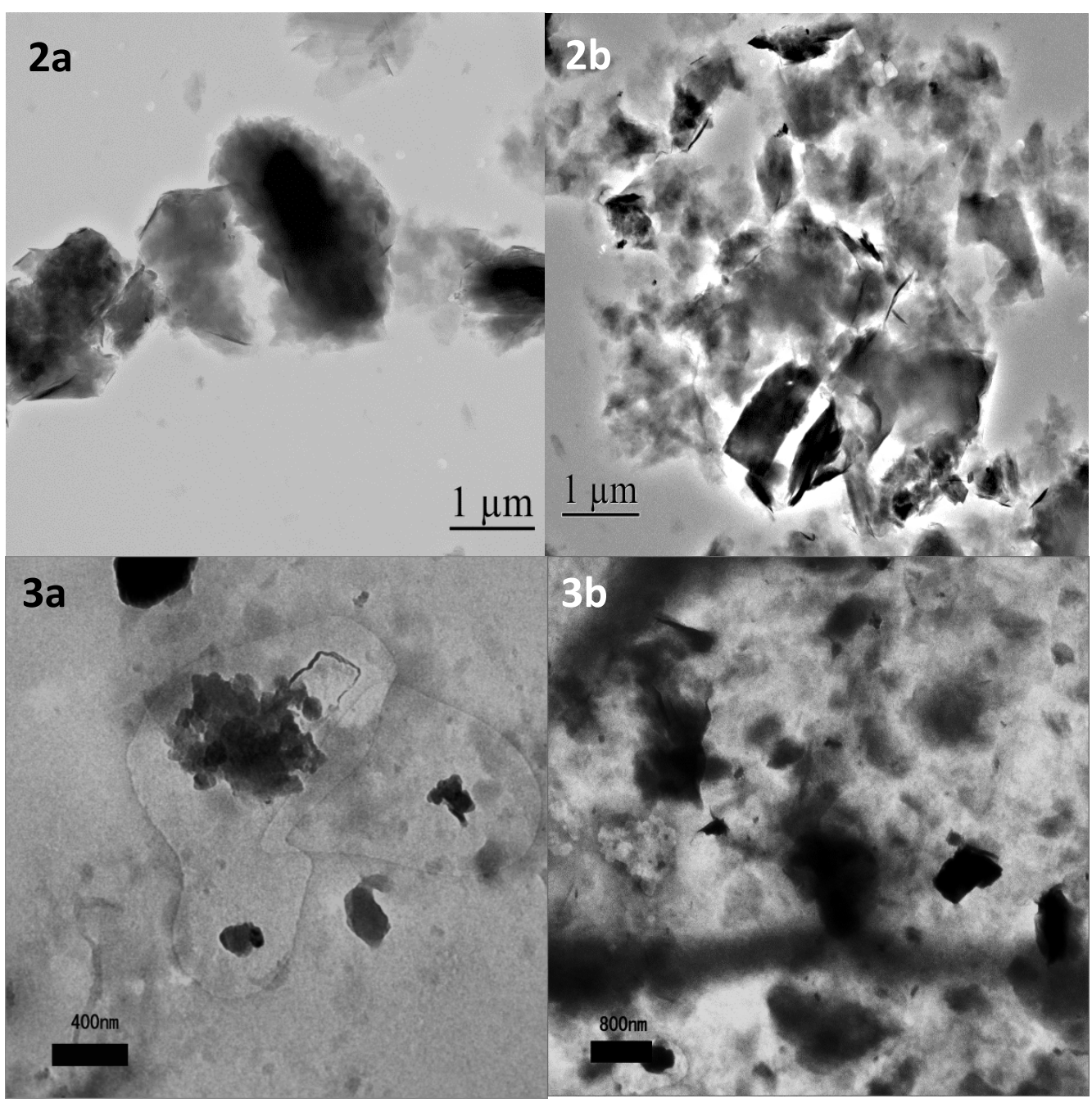

Fig. 2. Particle morphology is obscured by clay aggregates in conventional TEM images.

Fig. 3. Wet-cell TEM images revealing well-dispersed bentonite particles using simple EC method. Small particles with different shape can be seen with a larger distribution range. 